\title{
On the optimal parameter of the composite Laplacian quadratics function *
}

\author{
Fei Chen ${ }^{\mathrm{a}}$ and Gang Feng ${ }^{\mathrm{b}}$ \\ ${ }^{a}$ Department of Automation, Xiamen University, Xiamen, Fujian 361005, China \\ ${ }^{\mathrm{b}}$ Department of Mechanical and Biomedical Engineering, City University of Hong Kong, Kowloon, Hong Kong SAR, China
}

\begin{abstract}
Recently, the composite quadratic Lyapunov function has been extended to study of multi-agent systems, leading to the socalled composite Laplacian quadratics (CLQs) function. Compared with quadratic Lyapunov functions, the CLQs function can yield a larger convergence region and is particularly useful in stabilization of multi-agent systems with complex dynamics, such as differential inclusions. In the definition of the CLQs function, an optimal vector parameter plays a critical role in determining the value of the CLQs function and in constructing stabilization laws derived from the CLQs function. This paper focuses on the properties of the optimal parameter of the CLQs function. The uniqueness of the optimal parameter is established. A distributed computation approach is further proposed, which is useful in computing the optimal parameter. The robustness issue of the optimal parameter is also investigated for a multi-agent system described by linear differential inclusions. Finally, a numerical example is provided to validate the proposed theoretical results.
\end{abstract}

Key words: Composite Laplacian quadratics, multi-agent systems, optimality, distributed algorithm, robustness.

\section{Introduction}

Recently, the function of composite Laplacian quadratics is proposed for synthesis and analysis of multi-agent systems (Chen et al. (2015)), which can be seen as an extension of the composite quadratic function ( $\mathrm{Hu}$ and $\mathrm{Lin}$ (2004); Goebel et al. (2006)) to the study of multi-agent systems, where the Laplacian matrix is introduced to describe the communication graph among agents. The function is particularly useful in stabilization of multiagent systems whose dynamics are governed by linear differential inclusions (LDIs), which can be used to model a wide class of practical systems (Boyd et al. (1994)). It has been demonstrated that the CLQs function can yield a larger stabilization region, as compared to that given by quadratic Lyapunov functions ( $\mathrm{Hu}(2007))$. In addition, by the technique of global linearization in Liu (1968); Liu et al. (1971), a nonlinear time-varying system can be transformed into an LDI system, which suggests that the CLQs function can be employed to stabi-

\footnotetext{
* This work was supported in part by the National Science Foundation of China under grant 61473240 and the Research Grants Council of Hong Kong under grant CityU-11203714. Correspondence should be addressed to Fei Chen.

Email addresses: feichen@xmu.edu.cn (Fei Chen), megfeng@cityu.edu.hk (Gang Feng).
}

lize nonlinear time-varying systems as well (Boyd et al. (1994)). The function is used to design a nonlinear consensus algorithm for a multi-agent system (Chen et al. (2015)), for which consensus cannot be reached via linear algorithms designed under the quadratic stabilization framework. Because consensus is the basis for more involved cooperative control problems, the CLQs function has potential applications in other cooperative control problems of multi-agent systems such as distributed optimization (Nedic and Ozdaglar (2009); Sarlette and Sepulchre (2009)).

Construction of Lyapunov functions is one of the central tasks in study of multi-agent systems. Lyapunov functions are not only used for analysis of multi-agent systems, but also play a critical rule in controller synthesis of multi-agent systems. One type of quadratic function$\mathrm{s}$ that is commonly used is $V(x) \triangleq x^{T}(L \otimes P) x$, where $L$ is the Laplacian matrix of an undirected graph and $P$ is a positive definite matrix (Olfati-Saber and Murray (2004); Ren et al. (2007); Ren and Beard (2008)). Quadratic Lyapunov functions can also be designed for multi-agent systems under directed network topologies and various input or communication constrains (Meng et al. (2013); Meng and Lin (2014)). Although quadratic Lyapunov functions serve as a fundamental tool in 
stability analysis and control synthesis, their limitations have been revealed in some papers (Moreau (2005); Ooba (2003)). Therefore, some non-quadratic Lyapunov functions have been proposed as a complement of quadratic Lyapunov functions. The set-valued Lyapunov function

$$
V_{\text {conv }} \triangleq\left(\operatorname{conv}\left\{x_{1}, \ldots, x_{n}\right\}\right)^{n}
$$

is used in Moreau (2005) to analyze the convergence of a nonlinear multi-agent model, where $\operatorname{conv}\left\{x_{1}, \ldots, x_{n}\right\}$ denotes the convex hull and $(\cdot)^{n}$ denotes the Cartesian product. Different from the quadratic Lyapunov function, the set-valued Lyapunov function can be used to conclude that the state of the system converges to one equilibrium out of a continuum of equilibria. Finally, it is worth mentioning that other types of Lyapunov functions such as the max-min function $V_{\text {max }, \min } \triangleq \max \left\{x_{1}, \ldots, x_{n}\right\}-\min \left\{x_{1}, \ldots, x_{n}\right\}$ (Moreau (2004); Cao et al. (2010)) are also employed in the literature for multi-agent systems.

As an extension of the composite quadratic function, the CLQs function inherits a very good property of the composite quadratic function, that is, it is continuously differentiable. This property is particularly useful in constructing continuous control laws from the CLQs. Note that a continuous control law, if exists, is always preferable to a discontinuous control law due to various considerations, such as avoiding the chattering effect. The value of the CLQs function is normally determined by solving an optimization problem, whose solution is given in terms of a vector parameter. The optimal value of the vector parameter plays a critical role not only in determining the value of the CLQs function but also in designing the control law under the CLQs function. The challenge of determining the optimal parameter is that the parameter is time-varying and depends on the state of the system. This implies that global information of the multi-agent system is needed in determining the optimal parameter. This motivates the problem of designing a distributed algorithm for determining the optimal parameter. Moreover, the computation of the optimal parameter needs time, which is not negligible for multiagent systems where communication is involved. One possible way to solve the computational time issue is to use "out-dated" values of the optimal parameter. The question is what is the upper bound on the deviation of the "out-dated" values from the true value of the optimal parameter that can be employed while guaranteeing the stability of the resulting system. This motivates the robustness issue of the optimal parameter of the CLQs function in controller synthesis of multi-agent systems.

The contributions of this paper are stated as follows. First, the uniqueness of the optimal parameter of the CLQs function is established. Second, a distributed algorithm is proposed which plays an important role in computing the optimal parameter of the CLQs function and is necessary for the distributed implementation of the control algorithm designed via the CLQs function. Finally, the robustness issue of the optimal parameter is investigated for a multi-agent system described by LDIs. It is proved that if the calculation error of the optimal parameter is smaller than certain upper bound, then consensus of the resulting controlled multi-agent system can still be guaranteed even if the algorithm employs an "inaccurate" value of the optimal parameter.

The rest of the paper is organized as follows. In Section 2, some notations and mathematical preliminaries are introduced. The uniqueness result of the optimal parameter of the CLQs function is established in Section 3. Section 4 presents a distributed algorithm for computing the optimal parameter of the CLQs function. The robustness issue of the optimal parameter is discussed in Section 5. A simulation example is then given in Section 6 to verify the obtained result on the robustness of the optimal parameter. Finally, Section 7 concludes this paper and gives some future directions.

\section{Preliminaries}

Let $\mathbb{R}$ denote the set of all real numbers and $\mathbb{R}^{+}$the set of all positive real numbers. Let $\mathbb{R}^{n}$ denote the set of $n$ dimensional real vectors and $\mathbb{R}^{m \times n}$ the set of $m \times n$ real matrices. Let $I_{n} \in \mathbb{R}^{n \times n}$ be the $n$-dimensional identity matrix, $\mathbf{0}_{n} \in \mathbb{R}^{n}$ the vector with all zeroes, and $\mathbf{1}_{n} \in \mathbb{R}^{n}$ the vector with all ones. Let $\mathfrak{i}_{j} \in \mathbb{R}^{n}$ denote the vector with all zeroes, except that the $j$ th entry is one. The subscripts of $I_{n}, \mathbf{0}_{n}$, and $\mathbf{1}_{n}$ might be dropped if no confusion arises in the context. For a vector $x \in \mathbb{R}^{n}$, define $\|x\| \triangleq\left(\left|x_{1}\right|^{2}+\cdots+\left|x_{n}\right|^{2}\right)^{1 / 2}$ and let $\operatorname{diag}(x) \in$ $\mathbb{R}^{n \times n}$ be the diagonal matrix constructed from $x$ with the elements in the main diagonal being the elements of $x$. For a matrix $A \in \mathbb{R}^{m \times n},\|A\| \triangleq \sqrt{\rho\left(A A^{T}\right)}$ denotes its induced two-norm, where $A^{T}$ is the transpose and $\rho(\cdot)$ is the spectral radius. The term "if and only if" is abbreviated as "iff". Let $S$ be a compact convex set. A point $x_{0} \in S$ is an extreme point of $S$ if it cannot be represented as the convex combination of other points in $S$. A hyperplane $c^{T} x=k$ is a supporting hyperplane of $S$ at $x_{0} \in \partial S$ if $c^{T} x \leq k$ for all $x \in S$ and $c^{T} x_{0}=k$, where $\partial S$ denotes the boundary of $S$.

A graph is defined as $\mathcal{G} \triangleq(\mathcal{V}, \mathcal{E})$, where $\mathcal{V}$ is the set of nodes and $\mathcal{E} \subseteq \mathcal{V} \times \mathcal{V}$ the set of edges. A graph is simple if it does not contain self-loops, nor have multiple edges between two nodes. Graph $\mathcal{G}$ is undirected if, for all $u, v \in$ $\mathcal{V},(u, v) \in \mathcal{E} \Longleftrightarrow(v, u) \in \mathcal{E}$. In this paper, only simple and undirected graphs are considered. The order and size of $\mathcal{G}$ are denoted, respectively, by $n \triangleq|\mathcal{V}|$ and $m \triangleq|\mathcal{E}|$, where $|\cdot|$ denotes the number of elements in a set. A path from node $v_{1}$ to node $v_{k}$ is a sequence of nodes $v_{1}, \ldots, v_{k}$, such that for each $i, 1 \leq i \leq k-1,\left(v_{i}, v_{i+1}\right)$ is an edge. A graph is connected if there is a path from any node to 
any other node in the graph. Let $A \triangleq\left[a_{i j}\right] \in \mathbb{R}^{n \times n}$ be the adjacency matrix of $\mathcal{G}$. The degree of node $i$ is defined as $d_{i} \triangleq \sum_{j=1}^{n} a_{i j}$, and the degree matrix is defined as $D \triangleq \operatorname{diag}\left(\left[d_{1}, \ldots, d_{n}\right]\right) \in \mathbb{R}^{n \times n}$. The Laplacian matrix of $\mathcal{G}$ is then given by $L \triangleq D-A \in \mathbb{R}^{n \times n}$. It can be verified that the Laplacian matrix $L$ is positive semi-definite, and has a zero eigenvalue whose normalized eigenvector is $(1 / \sqrt{n}) \mathbf{1}_{n}$. Without loss of generality, let $0=\lambda_{1} \leq \lambda_{2} \leq$ $\cdots \leq \lambda_{n}$ denote the $n$ eigenvalues of $L$. Here, the secondsmallest eigenvalue $\lambda_{2}$ is called the algebraic connectivity of graph $\mathcal{G}$, which is related to the connectivity of graph $\mathcal{G}$ by the following lemma.

Lemma 1 (Godsil and Royle (2001)) The algebraic connectivity $\lambda_{2}>0$ iff $\mathcal{G}$ is a connected graph.

For a symmetric block matrix $\mathfrak{X}=\left[\begin{array}{cc}\mathfrak{A} & \mathfrak{B} \\ \mathfrak{B}^{T} & \mathfrak{C}^{\mathfrak{C}}\end{array}\right]$, its Schur complement is defined by $\mathfrak{A}-\mathfrak{B C} \mathfrak{C}^{-1} \mathfrak{B}^{T}$ if $\mathfrak{C}^{-1}$ exists. The positive definiteness (positive semi-definiteness) of the block matrix $\mathfrak{X}$ can be verified via its Schur complement.

Lemma 2 (Schur complement, Boyd et al. (1994)) For the symmetric matrix $\mathfrak{X}$, the following holds

- $\mathfrak{X}>0 \Longleftrightarrow \mathfrak{C}>0, \mathfrak{A}-\mathfrak{B} \mathfrak{C}^{-1} \mathfrak{B}^{T}>0$;

- $\mathfrak{X} \geq 0 \Longleftrightarrow \mathfrak{C}>0, \mathfrak{A}-\mathfrak{B C}^{-1} \mathfrak{B}^{T} \geq 0$.

Let $\mathfrak{A}(\mathfrak{x})$ be an invertible matrix depending on a real parameter $\mathfrak{x} \in \mathcal{X} \subset \mathbb{R}$. The following lemma shows how to calculate the derivative of the inverse matrix $\mathfrak{A}^{-1}$.

Lemma 3 (Horn and Johnson (2012)) Let $B$ be a matrix such that $\|B\|<1$, then the matrix $(I+B)$ is invertible, and $\left\|(I+B)^{-1}\right\| \leq \frac{1}{1-\|B\|}$.

\section{Uniqueness of the optimal parameter of the CLQs function}

In what follows, a formal definition of the CLQs function is first given. Let $Q_{j}=Q_{j}^{T} \in \mathbb{R}^{p \times p}, j=1, \ldots, n_{Q}$, be a family of positive definite matrices. Define

$$
\Gamma^{n_{Q}} \triangleq\left\{\gamma=\left[\gamma_{1}, \ldots, \gamma_{n_{Q}}\right]^{T}: \gamma_{j} \geq 0, \sum_{j=1}^{n_{Q}} \gamma_{j}=1\right\}
$$

The CLQs function is then defined as

$$
V(x) \triangleq \min _{\gamma \in \Gamma^{n_{Q}}} x^{T}\left[L \otimes\left(\sum_{j=1}^{n_{Q}} \gamma_{j} Q_{j}\right)^{-1}\right] x
$$

where $L \in \mathbb{R}^{n \times n}$ is the Laplacian matrix of a graph $\mathcal{G}$, and $x \in \mathbb{R}^{n p}$ denotes the state of the system. Throughout the paper, the following assumption on $\mathcal{G}$ is made.

Assumption 1 Graph $\mathcal{G}$ is undirected and connected.
The one-level set of the CLQs function is defined as

$$
O_{V} \triangleq\left\{x \in \mathbb{R}^{n p}: V(x) \leq 1\right\}
$$

For the function $x \mapsto x^{T}\left(L \otimes Q_{j}^{-1}\right) x$, its one-level set is denoted by $O_{L \otimes Q_{j}^{-1}} \triangleq\left\{x \in \mathbb{R}^{n p}: x^{T}\left(L \otimes Q_{j}^{-1}\right) x \leq 1\right\}$, where $Q_{j} \in \mathbb{R}^{p \times p}$ is the positive definite matrix defined in $(1)$.

When the system state $x$ is given, the value of the CLQs function can be determined by solving the optimization problem (1), whose solution is given in terms of the parameter $\gamma$. It is not difficult to see that the optimal value of $\gamma$ depends on the system state $x$. Specifically, let $\gamma^{*}(x)$ be the optimal $\gamma$ of the CLQs function, i.e.,

$$
\gamma^{*}(x) \triangleq \arg \min _{\gamma \in \Gamma^{n_{Q}}} x^{T}\left[L \otimes\left(\sum_{j=1}^{n_{Q}} \gamma_{j} Q_{j}\right)^{-1}\right] x
$$

When there exists no confusion, $\gamma^{*}(x)$ is simply written as $\gamma^{*}$ for notational convenience.

For the convenience of further analysis, define $O_{\tilde{c}} \triangleq\{\tilde{y} \in$ $\left.\mathbb{R}^{(n-1) p}:\left|\tilde{c}^{T} \tilde{y}\right| \leq 1\right\}$ for a vector $\tilde{c} \in \mathbb{R}^{(n-1) p}$, and let $O_{\tilde{\Lambda} \otimes Q_{j}^{-1}} \triangleq\left\{\tilde{y} \in \mathbb{R}^{(n-1) p}: \tilde{y}^{T}\left(\tilde{\Lambda} \otimes Q_{j}^{-1}\right) \tilde{y} \leq 1\right\}$, where $\tilde{\Lambda} \triangleq \operatorname{diag}\left(\left[\lambda_{2}, \ldots, \lambda_{n}\right]\right)$, The following result establishes the relationship between the one-level set $O_{\tilde{\Lambda} \otimes Q_{j}^{-1}}$ and the hyperplane $\tilde{c}^{T} \tilde{y}=1$, which will be employed to prove the uniqueness of $\gamma^{*}$.

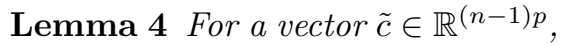

(i) $O_{\tilde{\Lambda} \otimes Q_{j}^{-1}} \subseteq O_{\tilde{c}}$ iff

$$
\tilde{c}^{T}\left(\tilde{\Lambda}^{-1} \otimes Q_{j}\right) \tilde{c} \leq 1
$$

(ii) The equality $\tilde{c}^{T}\left(\tilde{\Lambda}^{-1} \otimes Q_{j}\right) \tilde{c}=1$ holds iff $O_{\tilde{\Lambda} \otimes Q_{j}^{-1}}$ and the hyperplane $\tilde{c}^{T} \tilde{y}=1$ have a unique intersection at $\tilde{y}_{0}=\left(\tilde{\Lambda}^{-1} \otimes Q_{j}\right) \tilde{c}$. In particular, $\tilde{c}^{T} \tilde{y}<$ $\tilde{c}^{T} \tilde{y}_{0}=1$ for all $\tilde{y} \in O_{\tilde{\Lambda} \otimes Q_{j}^{-1}} \backslash\left\{\tilde{y}_{0}\right\}$, i.e., $\tilde{c}^{T} \tilde{y}=1$ is a supporting hyperplane of $O_{\tilde{\Lambda} \otimes Q_{j}^{-1}}$ at $\tilde{y}_{0}$.

PROOF. $O_{\tilde{\Lambda} \otimes Q_{j}^{-1}} \subseteq O_{\tilde{c}}$ holds iff the hyperplanes $\tilde{c}^{T} \tilde{y}= \pm 1$ lie completely outside the ellipsoid $O_{\tilde{\Lambda} \otimes Q_{j}^{-1}}$. That is,

$$
\min \left\{\tilde{y}^{T}\left(\tilde{\Lambda} \otimes Q_{j}^{-1}\right) \tilde{y}: \tilde{c}^{T} \tilde{y}=1\right\} \geq 1
$$

Note that the case for $\tilde{c}^{T} \tilde{y}=-1$ is already included in (4), because $\tilde{c}^{T} \tilde{y}=-1$ is equivalent to $\tilde{c}^{T}(-\tilde{y})=1$ and $(-\tilde{y})^{T}\left(\tilde{\Lambda} \otimes Q_{j}^{-1}\right)(-\tilde{y})=\tilde{y}^{T}\left(\tilde{\Lambda} \otimes Q_{j}^{-1}\right) \tilde{y}$. The left-hand 
side of (4) is an optimization problem with the optimization function $\tilde{y}^{T}\left(\tilde{\Lambda} \otimes Q_{j}^{-1}\right) \tilde{y}$ and the constraint $\tilde{c}^{T} \tilde{y}=1$, which can be solved by the following Lagrangian:

$$
f(\tilde{y}, \lambda) \triangleq \tilde{y}^{T}\left(\tilde{\Lambda} \otimes Q_{j}^{-1}\right) \tilde{y}+\lambda\left(1-\tilde{c}^{T} \tilde{y}\right)
$$

where $\lambda \in \mathbb{R}$ is the Lagrange multiplier. The stationary points of (5) must satisfy

$$
\begin{aligned}
& \frac{\partial f}{\partial \tilde{y}}=2\left(\tilde{\Lambda} \otimes Q_{j}^{-1}\right) \tilde{y}+\lambda(-\tilde{c})=0 \\
& \frac{\partial f}{\partial \lambda}=1-\tilde{c}^{T} \tilde{y}=0 .
\end{aligned}
$$

The solution to (6) is given by

$$
\begin{aligned}
\lambda & =2 \frac{1}{\tilde{c}^{T}\left(\tilde{\Lambda}^{-1} \otimes Q_{j}\right) \tilde{c}}, \\
\tilde{y} & =\frac{1}{\tilde{c}^{T}\left(\tilde{\Lambda}^{-1} \otimes Q_{j}\right) \tilde{c}}\left(\tilde{\Lambda}^{-1} \otimes Q_{j}\right) \tilde{c} .
\end{aligned}
$$

Thus, the minimum of $\tilde{y}^{T}\left(\tilde{\Lambda} \otimes Q_{j}^{-1}\right) \tilde{y}$ subject to the constraint $\tilde{c}^{T} \tilde{y}=1$ is given by $\tilde{y}^{T}\left(\tilde{\Lambda} \otimes Q_{j}^{-1}\right) \tilde{y}=\frac{1}{\tilde{c}^{T}\left(\tilde{\Lambda}^{-1} \otimes Q_{j}\right)} \tilde{c}$, which suggests that $O_{\tilde{\Lambda} \otimes Q_{j}^{-1}} \subseteq O_{\tilde{c}}$ iff (3) holds. The proof for (i) is thus completed.

The proof for the first part of (ii) " $\tilde{c}^{T}\left(\tilde{\Lambda}^{-1} \otimes Q_{j}\right) \tilde{c}=1$ iff $O_{\tilde{\Lambda} \otimes Q_{j}^{-1}}$ and the hyperplane $\tilde{c}^{T} \tilde{y}=1$ have a unique intersection at $\tilde{y}_{0}=\left(\tilde{\Lambda}^{-1} \otimes Q_{j}\right) \tilde{c}^{\prime \prime}$ is similar. The only difference is to replace the inequalities with the corresponding equalities. Note that the intersection is defined by $(7)$, where $\tilde{c}^{T}\left(\tilde{\Lambda}^{-1} \otimes Q_{j}\right) \tilde{c}=1$. Thus, the unique intersection point is given by $\tilde{y}_{0}=\left(\tilde{\Lambda}^{-1} \otimes Q_{j}\right) \tilde{c}$. Finally, $\tilde{c}^{T} \tilde{y}<\tilde{c}^{T} \tilde{y}_{0}=1$ for all $\tilde{y} \in O_{\tilde{\Lambda} \otimes Q_{j}-1} \backslash\left\{y_{0}\right\}$ follows immediately from the results of (i) and the first part of (ii).

Without loss of generality, assume that $\gamma_{j}^{*}>0$ for all $j=1, \ldots, n_{Q_{0}}$ and $\gamma_{j}^{*}=0$ for all $j=n_{Q_{0}}+1, \ldots, n_{Q}$, where $\gamma_{j}^{*}$ denotes the $j$ th element of $\gamma^{*}$ in (2). Moreover, define the following set

$$
\begin{aligned}
E_{j} & \triangleq \partial O_{V} \cap \partial O_{L \otimes Q_{j}^{-1}} \\
& =\left\{x \in \mathbb{R}^{n p}: V(x)=x^{T}\left(L \otimes Q_{j}^{-1}\right) x=1\right\}
\end{aligned}
$$

The following result gives the gradient of the CLQs function and shows that each $x \in \partial O_{V}$ can be written as a convex combination of the elements in $E_{j}$, $j=1, \ldots, n_{Q_{0}}$.

Lemma 5 (Chen et al. (2016)) For $x \in \partial O_{V}$, define
$Q\left(\gamma^{*}\right) \triangleq \sum_{j=1}^{n_{Q}} \gamma_{j}^{*} Q_{j}$ and $x_{j}^{*} \triangleq\left[I_{n} \otimes Q_{j} Q^{-1}\left(\gamma^{*}\right)\right] x$. Then,

$$
\begin{aligned}
\nabla V(x) & =\nabla V\left(x_{j}^{*}\right)=2\left(L \otimes Q_{j}^{-1}\right) x_{j}^{*}=2\left[L \otimes Q^{-1}\left(\gamma^{*}\right)\right] x \\
x_{j}^{*} & \in E_{j}, \quad j=1, \ldots, n_{Q_{0}}, \\
x & =\sum_{j=1}^{n_{Q_{0}}} \gamma_{j}^{*} x_{j}^{*} .
\end{aligned}
$$

Theorem 1 For a general choice of $Q_{j}, j=1, \ldots, n_{Q}$, $\gamma^{*}$ has a unique solution.

PROOF. Because $\gamma^{*}(k x)=\gamma^{*}(x)$ for $k \in \mathbb{R}$. Thus, in the following, we will focus on the uniqueness of $\gamma^{*}$ for $x \in \partial O_{V}$. Noting that the Laplacian matrix $L$ is symmetric, it can be diagonalized as follows:

$$
L=U^{T} \Lambda U
$$

where $U \in \mathbb{R}^{n \times n}$ is a unitary matrix satisfying $U^{T} U=$ $U U^{T}=I$, and $\Lambda \triangleq \operatorname{diag}\left(\left[\lambda_{1}, \ldots, \lambda_{n}\right]\right)$ with $\lambda_{i}$ denoting the $i$ th eigenvalue of the Laplacian matrix $L$. Define

$$
\tilde{y}=\left(\tilde{U} \otimes I_{p}\right) x
$$

where $\tilde{U} \in \mathbb{R}^{(n-1) \times n}$ is the matrix constructed from $U$, defined in (10), by removing the first row. Notice that $V(x)=\min _{\gamma \in \Gamma^{n_{Q}}} x^{T}\left[L \otimes\left(\sum_{j=1}^{n_{Q}} \gamma_{j} Q_{j}\right)^{-1}\right] x=$ $\min _{\gamma \in \Gamma^{n_{Q}}} \tilde{y}^{T}\left[\tilde{\Lambda} \otimes\left(\sum_{j=1}^{n_{Q}} \gamma_{j} Q_{j}\right)^{-1}\right] \tilde{y} \triangleq \tilde{V}(\tilde{y})$. Thus, $x \in O_{V}$ is equivalent to $\tilde{y} \in O_{\tilde{V}} \triangleq\{\tilde{y}: \tilde{V}(\tilde{y}) \leq 1\}$. Let $\tilde{E}_{j} \triangleq \partial O_{\tilde{\Lambda} \otimes Q_{j}^{-1}} \cap \partial O_{\tilde{V}}$. We first show that if for any $\tilde{y} \in \partial O_{\tilde{V}}$, the expression

$$
\tilde{y}=\sum_{j=1}^{n_{Q}} \gamma_{j}^{0} \tilde{y}_{j}^{0}, \quad \tilde{y}_{j}^{0} \in \tilde{E}_{j}, \quad \gamma^{0} \in \Gamma^{n_{Q}}
$$

is unique, then $\gamma^{*}$ is unique. This can be shown by the following contradiction statements. Suppose that there exist multiple $\gamma^{*}$. By multiplying both sides of (9) of Lemma 5 with $\tilde{U}^{T} \otimes I_{p}$, it follows that the expression (12) is also not unique. Thus, the uniqueness of $\gamma^{*}$ is guaranteed if the expression in (12) is unique.

In the following, a necessary and sufficient condition for the uniqueness of (12) is given. For any $\tilde{y} \in \partial O_{\tilde{V}}$, it must belong to a uniquely defined exposed face $\tilde{E}$ which is the intersection of $\partial O_{\tilde{V}}$ with certain supporting hyperplane $\tilde{c}^{T} \tilde{y}=1$. Following a similar proof to that of Property 1 of $\mathrm{Hu}$ and Lin (2004), it can be shown that the exposed face $\tilde{E}$ is a polygon with at most $n_{Q}$ vertices, which are the unique intersections of the supporting hyperplane $\tilde{c}^{T} \tilde{y}=1$ with the ellipsoids $\partial O_{\tilde{\Lambda} \otimes Q_{j}^{-1}}, j=1, \ldots, n_{Q}$, defined in Lemma 4 . Because $\tilde{y}$ and $\tilde{y}_{j}^{0}$ all belong to $\partial O_{\tilde{V}}$, if $\tilde{y}$ can be expressed as a convex combination of $\tilde{y}_{j}^{0}$, then 
$\tilde{y}_{j}^{0}$ must also belong to the same exposed face $\tilde{E}(\mathrm{Hu}$ and Lin (2004)). Thus, we only need to consider $\tilde{y}_{j}^{0}, j=$ $1, \ldots, n_{Q}$, that belong to the exposed face $\tilde{E}$. Without loss of generality, suppose that $\tilde{E}$ has intersections with the first $n_{0} \leq n_{Q}$ ellipsoids $\partial O_{\tilde{\Lambda} \otimes Q_{j}^{-1}}$. The uniqueness of (12) reduces to the uniqueness of

$$
\tilde{y}=\sum_{j=1}^{n_{0}} \gamma_{j}^{0} \tilde{y}_{j}^{0}, \quad \tilde{y}_{j}^{0} \in \tilde{E}_{j} \cap \tilde{E}, \quad \gamma^{0} \in \Gamma^{n_{Q}} .
$$

Note that a supporting hyperplane can have at most one intersection with an ellipsoid. Therefore, $\tilde{y}_{j}^{0}$ are uniquely defined in Lemma 4 , which indicates that the expression of (13) is unique iff $\tilde{y}_{j}^{0}, j=1, \ldots, n_{0}$, are linearly independent.

Finally, we will show that $\tilde{y}_{j}^{0} \in \tilde{E}_{j} \cap \tilde{E}, j=1, \ldots, n_{0}$, are linearly independent for a general choice of $Q_{j}$. To show this, it is equivalent to prove that for a general choice of $Q_{j}$ it is impossible for $\tilde{y}_{j}^{0}$ to be linearly dependent. $\tilde{y}_{j}^{0}$, $j=1, \ldots, n_{0}$, are linearly dependent iff either one of the following conditions holds:

(i) The number of the intersections $n_{0}$ is larger than the dimension of the state space of $\tilde{y}_{j}^{0}$, i.e., $n_{0}>$ $(n-1) p$.

(ii) $n_{0} \leq(n-1) p$ and $\tilde{y}_{j}^{0}, j=1, \ldots, n_{0}$, are linearly dependent.

By Lemma 4, the first condition holds iff the vector $\tilde{c} \in$ $\mathbb{R}^{(n-1) p}$ satifies

$$
\begin{gathered}
\tilde{c}^{T}\left(\tilde{\Lambda}^{-1} \otimes Q_{1}\right) \tilde{c}=1, \\
\vdots \\
\tilde{c}^{T}\left(\tilde{\Lambda}^{-1} \otimes Q_{n_{0}}\right) \tilde{c}=1, \\
n_{0}>(n-1) p .
\end{gathered}
$$

Note that (14) consists of $n_{0}$ equations with only $(n-1) p$ unknowns. Generally, there exits no solution $\tilde{c}$ satisfying (14). That is, the first condition cannot be satisfied for a general choice of $Q_{j}$. The second condition holds iff there exists $\tilde{c} \in \mathbb{R}^{(n-1) p}$ satisfying (14) for $n_{0}<(n-1) p$ and $\tilde{y}_{j}^{0}, j=1, \ldots, n_{0}$, are linearly dependent. Because $\tilde{y}_{j}^{0}$ are linearly dependent, there exist $\alpha_{j}, j=1, \ldots, n_{0}$, such that

$$
\begin{gathered}
\sum_{j=1}^{n_{0}} \alpha_{j} \tilde{y}_{j}^{0}=0, \\
\sum_{j=1}^{n_{0}} \alpha_{j}^{2}=1 .
\end{gathered}
$$

Note that there exists at least one nonzero $\alpha_{j}$ in (15), and if (15) holds for $\alpha_{j}$, then it also holds for all $k \alpha_{j}(k \in \mathbb{R})$. Thus, the existence of $\alpha_{j}$ satisfying (16) is guaranteed. There are $n_{0}+(n-1) p+1$ equations in (14)-(16) with only $n_{0}+(n-1) p$ unknowns, which indicates that (14)(16) have no solution for a general choice of $Q_{j}$. It can be concluded that the second condition cannot be satisfied for a general choice of $Q_{j}$, and then (13) is unique. The proof is thus completed.

\section{Distributed computation of the optimal pa- rameter of the CLQs function}

In this section, a distributed algorithm for computing the optimal parameter $\gamma^{*}$ is proposed. Because the network $\mathcal{G}$ is undirected, the Laplacian matrix $L$ can be written as $L=E^{T} E$, where $E \in \mathbb{R}^{m \times n}$ is the incidence matrix of $\mathcal{G}$. The function of composite Laplacian quadratics can be written as

$$
\begin{aligned}
V(x) & =\min _{\gamma \in \Gamma^{n_{Q}}} x^{T}\left[E^{T} E \otimes\left(\sum_{q=1}^{n_{Q}} \gamma_{q} Q_{q}\right)^{-1}\right] x \\
& =\min _{\gamma \in \Gamma^{n_{Q}}} x^{T}\left(E^{T} \otimes I_{p}\right)\left[I_{m} \otimes\left(\sum_{q=1}^{n_{Q}} \gamma_{q} Q_{q}\right)^{-1}\right]\left(E \otimes I_{p}\right) x \\
& =\min _{\gamma \in \Gamma^{n_{Q}}} \sum_{(i, j) \in \mathcal{E}}\left(x_{i}-x_{j}\right)^{T}\left(\sum_{q=1}^{n_{Q}} \gamma_{q} Q_{q}\right)^{-1}\left(x_{i}-x_{j}\right) .
\end{aligned}
$$

With a slight abuse of notation, let $(i, j)$ denote the $k$ th edge. Eq. (17) can be rewritten as

$$
\begin{aligned}
V(x)= & \min _{\gamma \in \Gamma^{n_{Q}}}\left\{\sum_{k=1}^{m} \alpha_{k}: \alpha_{k}=\left(x_{i}-x_{j}\right)^{T}\left(\sum_{q=1}^{n_{Q}} \gamma_{q} Q_{q}\right)^{-1}\right. \\
& \left.\left(x_{i}-x_{j}\right)\right\} \\
= & \min _{\gamma \in \Gamma^{n_{Q}}}\left\{\sum_{k=1}^{m} \alpha_{k}: \alpha_{k} \geq\left(x_{i}-x_{j}\right)^{T}\left(\sum_{q=1}^{n_{Q}} \gamma_{q} Q_{q}\right)^{-1}\right. \\
& \left.\left(x_{i}-x_{j}\right)\right\} .
\end{aligned}
$$

Remark 1 In (19), we add ">" to the equality of (18). This will not affect the definition of $V(x)$, as shown as follows. Suppose there exists a set $\mathcal{K} \subseteq\{1, \ldots, m\}$ such that $\alpha_{k}>\left(x_{i}-x_{j}\right)^{T}\left(\sum_{q=1}^{n_{Q}} \gamma_{q} Q_{q}\right)^{-1}\left(x_{i}-x_{j}\right)$ for $k \in \mathcal{K}$. Then, one can define $\alpha_{k}^{\prime}=\alpha_{k}$ for $k \notin \mathcal{K}$ and $\alpha_{k}^{\prime}=\left(x_{i}-x_{j}\right)^{T}\left(\sum_{q=1}^{n_{Q}} \gamma_{q} Q_{q}\right)^{-1}\left(x_{i}-x_{j}\right)$ for $k \in \mathcal{K}$. It is straightforward to obtain that $\sum_{k=1}^{m} \alpha_{k}^{\prime}<\sum_{k=1}^{m} \alpha_{k}$, which indicates that those $\sum_{k=1}^{m} \alpha_{k}$ with $\alpha_{k}>\left(x_{i}-\right.$ $\left.x_{j}\right)^{T}\left(\sum_{q=1}^{n_{Q}} \gamma_{q} Q_{q}\right)^{-1}\left(x_{i}-x_{j}\right)$ will not be the minimum. The above definition can be extended to the case of undirected weighted graphs by incorporating edge weights in (19).

Due to Lemma 2, an equivalent definition of $V(x)$ can 


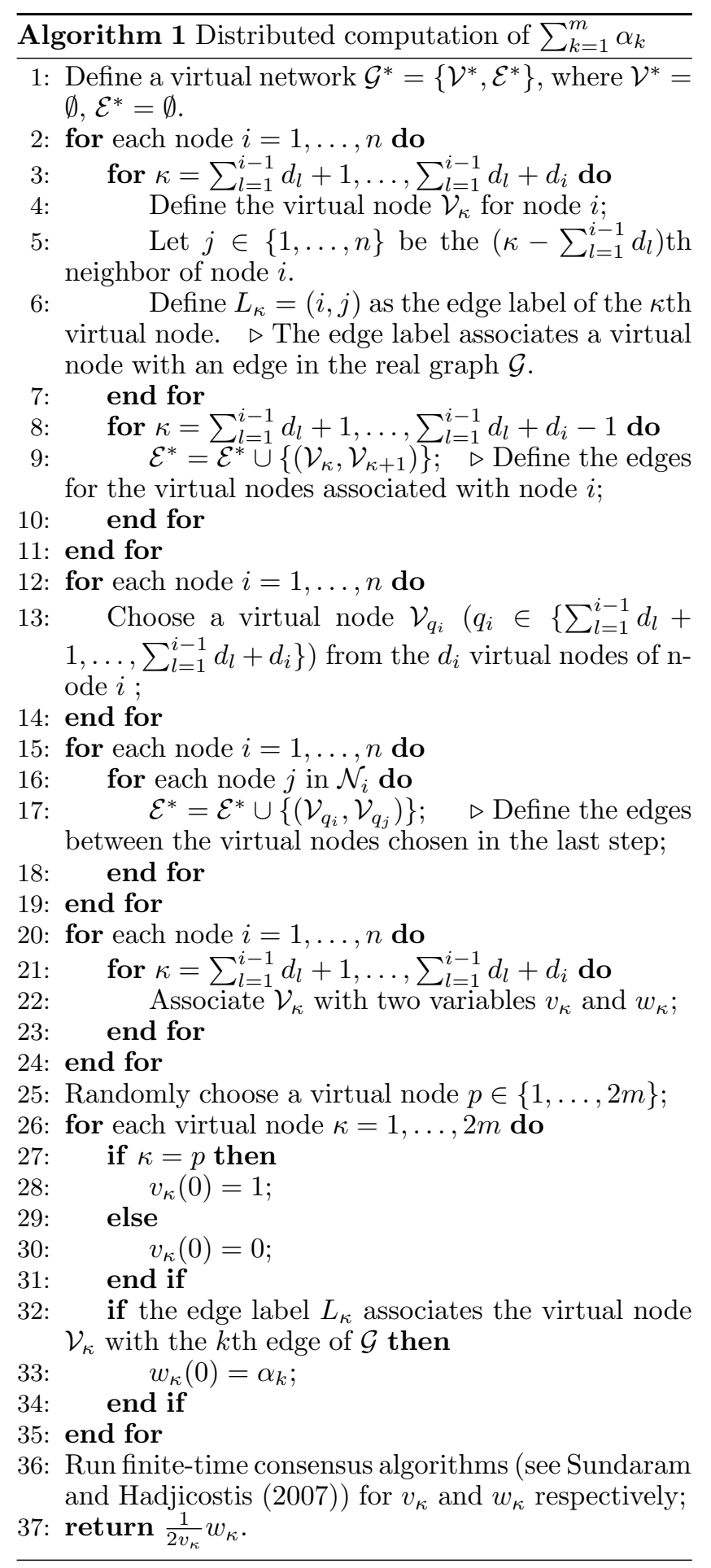

be given by

$$
\begin{aligned}
& V(x)=\min _{\alpha, \gamma} \sum_{k=1}^{m} \alpha_{k}, \\
& \text { s.t. }\left[\begin{array}{cc}
\alpha_{k} & \left(x_{i}-x_{j}\right)^{T} \\
\left(x_{i}-x_{j}\right) & \sum_{q=1}^{n_{Q}} \gamma_{q} Q_{q}
\end{array}\right] \geq 0, \\
& \sum_{q=1}^{n_{Q}} \gamma_{q}=1, \quad \gamma_{q} \geq 0 .
\end{aligned}
$$

Eqs. (20)-(22) is a typical optimization problem with linear matrix inequality constraints, where the optimization variables are $\gamma=\left[\gamma_{1}, \ldots, \gamma_{n_{Q}}\right]^{T}$ and $\alpha \triangleq\left[\alpha_{1}, \ldots, \alpha_{m}\right]^{T}$. The matrices $Q_{q}$ are given constant matrices for (20)-(22). If $m$ is not large, then the optimization problem (20)-(22) can be solved with various numerical techniques presented in Boyd and Vandenberghe (2004) in a centralized way. If $m$ is large, then a distributed optimization algorithm is preferable. In the following, we first present an algorithm which is used to compute the cost function $\sum_{k=1}^{m} \alpha_{k}$ in a distributed way. The procedure of the algorithm is described in Algorithm 1.

In Algorithm 1, Line 1 initializes the virtual graph $\mathcal{G}^{*}$. Lines 2-11 define the virtual nodes of the virtual graph, where each (real) node of $\mathcal{G}$ corresponds to $d_{i}$ virtual nodes in $\mathcal{G}^{*}$, and the edges between the virtual nodes associated with the same node. The time complexity for lines $2-11$ is $O\left(n \times\left(\max _{i} d_{i}\right)\right)$ which is less than $O\left(n^{2}\right)$. Lines $12-14$ choose a virtual node $\mathcal{V}_{q_{i}}$ from the virtual nodes associated with node $i$, whose complexity is given by $O(n)$. Lines 15-19 define the edges among the virtual nodes associated with different (real) nodes. The complexity is given by $O\left(n \times\left(\max _{i} d_{i}\right)\right)$, which is again less than $O\left(n^{2}\right)$. The construction of the virtual graph is then completed. Lines 20-35 associate each virtual node with two variables $v_{\kappa}$ and $w_{\kappa}$, and initialize these variables, whose complexity is $O\left(n^{2}\right)$ by using the fact that $m \leq \frac{1}{2} n(n-1)$. Line 36 runs the finite-time consensus algorithm for $v_{\kappa}$ and $w_{\kappa}$, respectively, over the virtual graph $\mathcal{G}^{*}$. The finite-time consensus algorithm stops after at most $d_{c}$ steps, where $d_{c}$ denotes the degree of the minimal polynomial of the weight matrix associated with the consensus algorithm (see Sundaram and Hadjicostis (2007) for the details). Since $d_{c}$ is upper bounded by the order of the virtual graph $2 m$ and $m \leq \frac{1}{2} n(n-1)$, the time complexity for line 36 is $O\left(n^{2}\right)$. In summary, we conclude that the time complexity for Algorithm 1 is $O\left(n^{2}\right)$. According to the well-known results for consensus (Jadbabaie et al. (2003)), it follows that $v_{\kappa} \rightarrow(1 / 2 m)$ and $w_{\kappa} \rightarrow \frac{\sum_{k=1}^{m} \alpha_{k}}{m}$. The function $\sum_{k=1}^{m} \alpha_{k}$ is then given by $\frac{1}{2 v_{\kappa}} w_{\kappa}$. If the real network $\mathcal{G}$ is connected, it can be verified that the virtual network must also be connected. Note that, in $\mathcal{G}^{*}$, two virtual nodes can communicate only if they are associated with the same real node or they are associated with two real nodes which are connected in $\mathcal{G}$. Thus, Algorithm 1 only uses local information and is distributed.

To solve the optimization problem (20)-(22), we first consider the unconstrained optimization problem (20) by omitting the two constraints (21) and (22). Here, the cost function is given by $\sum_{k=1}^{m} \alpha_{k}$. Suppose that we use the gradient descent method (Boyd and Vandenberghe (2004)) to solve (20). At each step, we need to compute $\sum_{k=1}^{m} \alpha_{k}$ (used in determining the step size) and its gradient (the search direction). The gradient of the 
cost function $\sum_{k=1}^{m} \alpha_{k}$ with respect to $\alpha_{p}, p=1, \ldots, m$, is given by $\frac{\partial \sum_{k=1}^{m} \alpha_{k}}{\partial \alpha_{p}}=1$, which is local information. The value of $\sum_{k=1}^{m} \alpha_{k}$ can be computed by Algorithm 1. Hence, Algorithm 1 is run during each step of the gradient descent algorithm whenever the cost function $\sum_{k=1}^{m} \alpha_{k}$ is computed. In this way, the unconstrained optimization problem (20) can be solved in a distributed way. To deal with the constraints (21) and (22), we can construct the Lagrange dual function to transform the constrained optimization problem (20)-(22) into an unconstrained optimization problem, which can then be solved similarly.

Remark 2 There are some nontrivial differences between the optimization problem (20)-(22) and the works on distributed optimization of multi-agent systems (Zhu and Martinez (2012); Nedic and Ozdaglar (2009); Notarstefano and Bullo (2011)). First, the optimization problem (20)-(22) is a semi-definite program, where the constraints are given in the form of linear matrix inequalities, while most works on distributed optimization of multi-agent systems consider constraints that are scalarvalued inequalities. Second, the optimal decision variables of $\alpha_{k}$ in (20)-(22) might have different values, while in Zhu and Martinez (2012); Nedic and Ozdaglar (2009) the optimal decision variables have the same value, i.e., a consensus is reached on the decision variables. Despite the above nontrivial differences, it is still possible to borrow some ideas from the existing works on distributed optimization to solve the problem (20)-(22). For instance, suitable value function can be constructed such that the optimization problem (20)-(22) can be transcribed into an abstract program (Carlone et al. (2014)). In this way, the constraints consensus algorithm developed in Notarstefano and Bullo (2011) can be used to solve the optimization problem.

\section{Robustness of the optimal parameter $\gamma^{*}$ for linear differential inclusions}

In this section, we consider a multi-agent system with the following LDIs dynamics:

$$
\dot{x}_{i}=\sum_{k=1}^{n_{s}} \tau_{k}(t)\left(A_{k} x_{i}+B_{k} u_{i}\right), \quad i=1, \ldots, n,
$$

where $x_{i} \in \mathbb{R}^{p}$ is the state, $u_{i} \in \mathbb{R}^{q}$ the control input to be designed, $A_{k} \in \mathbb{R}^{p \times p}$ and $B_{k} \in \mathbb{R}^{p \times q}$ two constant matrices, and $n_{s}$ the number of linear systems $\left(A_{k}, B_{k}\right)$ in the LDIs dynamics. The parameters $\tau_{k}(t)$ satisfy $\tau_{k}(t) \geq 0$, and $\sum_{k=1}^{n_{s}} \tau_{k}(t)=1$. Note that $\tau_{k}(t)$ are time-varying and their values are unknown. The LDI model (23) can be used to describe a wide variety of practical systems (Boyd et al. (1994)). In addition, using the technique of global linearization, a nonlinear time-varying system can be transformed into LDIs, which indicates that (23) might find applications in control synthesis and analysis of nonlinear time-varying systems. Define $x \triangleq\left[x_{1}^{T}, \ldots, x_{n}^{T}\right]^{T} \in \mathbb{R}^{n p}$ and $u \triangleq$ $\left[u_{1}^{T}, \ldots, u_{n}^{T}\right]^{T} \in \mathbb{R}^{n q}$. Eq. (23) can be written in a vector form as

$$
\dot{x}=\sum_{k=1}^{n_{s}} \tau_{k}(t)\left[\left(I_{n} \otimes A_{k}\right) x+\left(I_{n} \otimes B_{k}\right) u\right] .
$$

To stabilize the LDIs system (23), the following bilinear matrix inequality (BMI) assumption is needed (Chen et al. (2015); $\mathrm{Hu}(2007))$.

Assumption 2 There exist $\beta>0, Y_{j} \in \mathbb{R}^{q \times p}$, and $\eta_{j k l} \geq 0, k=1, \ldots, n_{s}, j, l=1, \ldots, n_{Q}$, such that for all $i=2, \ldots, n$,

$$
\begin{aligned}
& Q_{j} A_{k}^{T}+A_{k} Q_{j}+\lambda_{i} Y_{j}^{T} B_{k}^{T}+\lambda_{i} B_{k} Y_{j} \\
& \leq \sum_{l=1}^{n_{Q}} \eta_{j k l}\left(Q_{l}-Q_{j}\right)-\beta Q_{j}, \quad \forall j, k,
\end{aligned}
$$

where $Q_{j}$ are the positive definite matrices defined in (1), and $\lambda_{i}, i=2, \ldots, n$, are the $n-1$ eigenvalues of the Laplacian matrix $L$.

Using Assumption 1 and Lemma 1, it follows that $\lambda_{i}$ are all positive. BMIs like (25) can be solved by the Matlab toolbox PENlab.

In Chen et al. (2015), a control algorithm is designed for the consensus of (24), where the control input is given by

$$
u=\left[L \otimes F\left(\gamma^{*}\right)\right] x,
$$

where

$$
F\left(\gamma^{*}\right) \triangleq\left(\sum_{j=1}^{n_{Q}} \gamma_{j}^{*} Y_{j}\right)\left(\sum_{j=1}^{n_{Q}} \gamma_{j}^{*} Q_{j}\right)^{-1}
$$

with $Y_{j}$ defined in Assumption 2. It is worth mentioning that the optimal parameter $\gamma^{*}$ in (26) depends on the system state $x$. The closed-loop system is then given by $\dot{x}=\sum_{k=1}^{n_{s}} \tau_{k}(t)\left[\left(I_{n} \otimes A_{k}\right) x+\left(I_{n} \otimes B_{k}\right)\left[L \otimes F\left(\gamma^{*}\right)\right] x\right]$. It has been proved in Chen et al. (2015) that if Assumptions 1 and 2 hold, then the system can reach a consensus exponentially.

Remark 3 The control law (26) can be rewritten as $u_{i}=$ $\sum_{j \in \mathcal{N}_{i}} F\left(\gamma^{*}\right)\left(x_{i}-x_{j}\right)$, where $u_{i}$ is the control input of agent $i$. The state information $x_{i}$ and $x_{j}\left(j \in \mathcal{N}_{i}\right)$ is local information. The feedback gain matrix $F\left(\gamma^{*}\right)$ depends on $Y_{j}, Q_{j}$, and $\gamma^{*}$, where the matrices $Y_{j}$ and $Q_{j}$ can be computed in a distributed manner from the BMIs (25). The optimal parameter $\gamma^{*}$ can also be computed in a distributed way by the distributed algorithm proposed in Section 4. Thus, the control law (26) can be implemented in a distributed manner. 
In $(26)$, the exact value of the optimal parameter $\gamma^{*}$ is employed. However, under some circumstances, only the "approximate" value of $\gamma^{*}$ can be obtained. For example, when computation time of the optimal $\gamma^{*}$ cannot be neglected, we might be able to use only the "out-dated" information of $\gamma^{*}$. Or when there exists a measurement noise, we can only use the noisy value of $\gamma^{*}$. If $\gamma^{*}$ cannot be obtained accurately, the robustness issue of $\gamma^{*}$ arises, which is considered in this section.

Let $\gamma_{e}^{*}=\left[\gamma_{e, 1}^{*}, \ldots, \gamma_{e, n_{Q}}^{*}\right]^{T} \in \Gamma^{n_{Q}}$ be the "approximate" value of $\gamma^{*}$, which is actually employed in the control inputs (26). Define the following error vector $e^{*}=\left[e_{1}^{*}, \ldots, e_{n_{Q}}^{*}\right]^{T} \triangleq \gamma_{e}^{*}-\gamma^{*}$, which measures the bias between $\gamma_{e}^{*}$ and $\gamma^{*}$. The following boundedness assumption on $e^{*}$ is used in this section.

Assumption 3 There exists $\bar{e} \in \mathbb{R}^{+}$such that $\left|e_{j}^{*}\right|<\bar{e}$ for all $j=1, \ldots, n_{Q}$.

Using the parameter $\gamma_{e}^{*}$, the closed-loop system becomes

$$
\dot{x}=\sum_{k=1}^{n_{s}} \tau_{k}(t)\left\{\left(I_{n} \otimes A_{k}\right) x+\left(I_{n} \otimes B_{k}\right)\left[L \otimes F\left(\gamma_{e}^{*}\right)\right] x\right\} .
$$

Let $a \triangleq n_{Q}\left[\max _{j} \sqrt{\lambda_{\max }\left(Y_{j}^{T} Y_{j}\right)}\right]\left[\min _{j} \lambda_{\min }\left(Q_{j}\right)\right]^{-1}$, $b \triangleq n_{Q}\left[\min _{j} \lambda_{\min }\left(Q_{j}\right)\right]^{-1}\left[\max _{j} \lambda_{\max }\left(Q_{j}\right)\right], c \triangleq n_{Q} \times$ $\left[\max _{j} \lambda_{\max }\left(Q_{j}\right)\right]\left[\min _{j} \lambda_{\min }\left(Q_{j}\right)\right]^{-2}\left[\max _{j} \sqrt{\lambda_{\max }\left(Y_{j}^{T} Y_{j}\right)}\right]$, and $d \triangleq 2 \lambda_{n}\left[\min _{j} \lambda_{\min }\left(Q_{j}\right)\right]^{-1}\left[\max _{k} \sqrt{\lambda_{\max }\left(B_{k}^{T} B_{k}\right)}\right] \times$ $\left[\min _{j} \lambda_{\min }\left(Q_{j}^{-1}\right)\right]^{-1}$. The main result of this section is established in the following theorem, which shows that the optimal parameter has certain robustness property.

Theorem 2 For the system (28) employing the inaccurate parameter $\gamma_{e}^{*}$, if Assumptions 1-3 hold, and $\bar{e}<$ $\frac{\beta}{\beta b+a d+c d}$, then the system (28) can still reach a consensus exponentially.

PROOF. In the following, the term $F\left(\gamma_{e}^{*}\right)$ is first analyzed. According to the definition of $(27)$ and $\gamma_{e}^{*}=$ $\gamma^{*}+e^{*}$,

$$
\begin{aligned}
F\left(\gamma_{e}^{*}\right)= & \left(\sum_{j=1}^{n_{Q}} \gamma_{j}^{*} Y_{j}+\sum_{j=1}^{n_{Q}} e_{j}^{*} Y_{j}\right) \\
& \times\left(\sum_{j=1}^{n_{Q}} \gamma_{j}^{*} Q_{j}+\sum_{j=1}^{n_{Q}} e_{j}^{*} Q_{j}\right)^{-1},
\end{aligned}
$$

where $\gamma_{j}^{*}$ and $e_{j}^{*}$ denote respectively the $j$ th entry of $\gamma^{*}$ and $e^{*}$. The inverse $\left(\sum_{j=1}^{n_{Q}} \gamma_{j}^{*} Q_{j}+\sum_{j=1}^{n_{Q}} e_{j}^{*} Q_{j}\right)^{-1}$ can be rewritten as

$$
\begin{aligned}
& \left(\sum_{j=1}^{n_{Q}} \gamma_{j}^{*} Q_{j}+\sum_{j=1}^{n_{Q}} e_{j}^{*} Q_{j}\right)^{-1} \\
= & \left\{\left(\sum_{j=1}^{n_{Q}} \gamma_{j}^{*} Q_{j}\right)\left[I+\left(\sum_{j=1}^{n_{Q}} \gamma_{j}^{*} Q_{j}\right)^{-1}\left(\sum_{j=1}^{n_{Q}} e_{j}^{*} Q_{j}\right)\right]\right\}^{-1} \\
= & {\left[I+\left(\sum_{j=1}^{n_{Q}} \gamma_{j}^{*} Q_{j}\right)^{-1}\left(\sum_{j=1}^{n_{Q}} e_{j}^{*} Q_{j}\right)\right]^{-1}\left(\sum_{j=1}^{n_{Q}} \gamma_{j}^{*} Q_{j}\right)^{-1}, }
\end{aligned}
$$

provided that $\left[I+\left(\sum_{j=1}^{n_{Q}} \gamma_{j}^{*} Q_{j}\right)^{-1}\left(\sum_{j=1}^{n_{Q}} e_{j}^{*} Q_{j}\right)\right]^{-1}$ and $\left(\sum_{j=1}^{n_{Q}} \gamma_{j}^{*} Q_{j}\right)^{-1}$ exist. The inverse $\left(\sum_{j=1}^{n_{Q}} \gamma_{j}^{*} Q_{j}\right)^{-1}$ exists since $\left(\sum_{j=1}^{n_{Q}} \gamma_{j}^{*} Q_{j}\right)$ is positive definite. The existence of the inverse $\left[I+\left(\sum_{j=1}^{n_{Q}} \gamma_{j}^{*} Q_{j}\right)^{-1}\left(\sum_{j=1}^{n_{Q}} e_{j}^{*} Q_{j}\right)\right]^{-1}$ can be derived as follows. Because

$$
\left\|\left(\sum_{j=1}^{n_{Q}} e_{j}^{*} Q_{j}\right)\right\| \leq \bar{e}\left(\sum_{j=1}^{n_{Q}}\left\|Q_{j}\right\|\right) \leq \bar{e} n_{Q}\left[\max _{j} \lambda_{\max }\left(Q_{j}\right)\right]
$$

and

$$
\begin{aligned}
\left\|\left(\sum_{j=1}^{n_{Q}} \gamma_{j}^{*} Q_{j}\right)^{-1}\right\| & =\lambda_{\max }\left[\left(\sum_{j=1}^{n_{Q}} \gamma_{j}^{*} Q_{j}\right)^{-1}\right]=\lambda_{\min }^{-1}\left[\left(\sum_{j=1}^{n_{Q}} \gamma_{j}^{*} Q_{j}\right)\right] \\
& \leq\left[\min _{j} \lambda_{\min }\left(Q_{j}\right)\right]^{-1}
\end{aligned}
$$

it follows that

$$
\begin{aligned}
& \left\|\left(\sum_{j=1}^{n_{Q}} \gamma_{j}^{*} Q_{j}\right)^{-1}\left(\sum_{j=1}^{n_{Q}} e_{j}^{*} Q_{j}\right)\right\| \\
\leq & {\left[\min _{j} \lambda_{\min }\left(Q_{j}\right)\right]^{-1} \bar{e} n_{Q}\left[\max _{j} \lambda_{\max }\left(Q_{j}\right)\right] } \\
= & b \bar{e} \quad \\
< & \frac{\beta b}{\beta b+a d+c d}<1 .
\end{aligned}
$$

It is noted that Eq. (33) is due to Eqs. (31) and (32), Eq. (34) is due to the definition of $b$, and Eq. (35) is due to the assumption $\bar{e}<\frac{\beta}{\beta b+a d+c d}$ in Theorem 2 and the fact that $a, b, c, d$, and $\beta$ are all positive constants. Thus, it follows from Lemma 3 that the inverse $\left[I+\left(\sum_{j=1}^{n_{Q}} \gamma_{j}^{*} Q_{j}\right)^{-1}\left(\sum_{j=1}^{n_{Q}} e_{j}^{*} Q_{j}\right)\right]^{-1}$ in (30) exists. In addition, it can be further written as

$$
\left[I+\left(\sum_{j=1}^{n_{Q}} \gamma_{j}^{*} Q_{j}\right)^{-1}\left(\sum_{j=1}^{n_{Q}} e_{j}^{*} Q_{j}\right)\right]^{-1}=I-\Xi
$$

where $\Xi \triangleq\left[I+\left(\sum_{j=1}^{n_{Q}} \gamma_{j}^{*} Q_{j}\right)^{-1}\left(\sum_{j=1}^{n_{Q}} e_{j}^{*} Q_{j}\right)\right]^{-1} \times$ $\left(\sum_{j=1}^{n_{Q}} \gamma_{j}^{*} Q_{j}\right)^{-1}\left(\sum_{j=1}^{n_{Q}} e_{j}^{*} Q_{j}\right)$. Substituting (36) into 
(30) gives $\left(\sum_{j=1}^{n_{Q}} \gamma_{j}^{*} Q_{j}+\sum_{j=1}^{n_{Q}} e_{j}^{*} Q_{j}\right)^{-1}=\left(\sum_{j=1}^{n_{Q}} \gamma_{j}^{*} Q_{j}\right)^{-1}-$ Note that $F\left(\gamma^{*}\right)=Y_{j} Q_{j}^{-1}$ due to $\gamma^{*}=\mathfrak{i}_{j}$. Using (37), $\Xi\left(\sum_{j=1}^{n_{Q}} \gamma_{j}^{*} Q_{j}\right)^{-1}$. Therefore, (29) can be rewritten as

$$
F\left(\gamma_{e}^{*}\right)=\left(\sum_{j=1}^{n_{Q}} \gamma_{j}^{*} Y_{j}\right)\left(\sum_{j=1}^{n_{Q}} \gamma_{j}^{*} Q_{j}\right)^{-1}+\Delta
$$

where $\Delta$ is defined by $\Delta \triangleq\left(\sum_{j=1}^{n_{Q}} e_{j}^{*} Y_{j}\right)\left(\sum_{j=1}^{n_{Q}} \gamma_{j}^{*} Q_{j}\right)^{-1}-$ $\left(\sum_{j=1}^{n_{Q}} e_{j}^{*} Y_{j}\right) \Xi\left(\sum_{j=1}^{n_{Q}} \gamma_{j}^{*} Q_{j}\right)^{-1}-\left(\sum_{j=1}^{n_{Q}} \gamma_{j}^{*} Y_{j}\right) \Xi \times$ $\left(\sum_{j=1}^{n_{Q}} \gamma_{j}^{*} Q_{j}\right)^{-1}$. It can be verified that

$$
\begin{aligned}
\left\|\left(\sum_{j=1}^{n_{Q}} e_{j}^{*} Y_{j}\right)\right\| & \leq \bar{e}\left(\sum_{j=1}^{n_{Q}}\left\|Y_{j}\right\|\right) \\
& \leq \bar{e} n_{Q}\left[\max _{j} \sqrt{\lambda_{\max }\left(Y_{j}^{T} Y_{j}\right)}\right]
\end{aligned}
$$

By Lemma 3, it follows that

$$
\begin{aligned}
& \left\|\left[I+\left(\sum_{j=1}^{n_{Q}} \gamma_{j}^{*} Q_{j}\right)^{-1}\left(\sum_{j=1}^{n_{Q}} e_{j}^{*} Q_{j}\right)\right]^{-1}\right\| \\
\leq & \frac{1}{1-\left\|\left(\sum_{j=1}^{n_{Q}} \gamma_{j}^{*} Q_{j}\right)^{-1}\left(\sum_{j=1}^{n_{Q}} e_{j}^{*} Q_{j}\right)\right\|} \\
\leq & \frac{1}{1-b \bar{e}},
\end{aligned}
$$

where the last inequality is due to (34). Using (31), (32), and (38)-(40), an upper bound on $\Delta$ is given by $\|\Delta\| \leq$ $\bar{e} \bar{\delta}$, where $\bar{\delta}$ is a constant defined as $\bar{\delta} \triangleq a+\bar{e} \frac{a b}{1-b \bar{e}}+\frac{c}{1-b \bar{e}}$.

In the following, the convergence of the system (28) is proved via the CLQs function. First, consider the case $x \in E_{j}$ for some fixed $j=1, \ldots, n_{Q}$, where $E_{j}$ is defined in (8). Using the definition of $E_{j}$ and $\gamma^{*}$, it follows that $\gamma^{*}=\mathfrak{i}_{j} \quad$ (or equivalently $\gamma_{j}^{*}=1$ and $\gamma_{p}^{*}=0$ for $p \neq j$ ). By Lemma 5 , the gradient of $V(x)$ is given by

$$
\nabla V(x)=2\left[L \otimes Q^{-1}\left(\gamma^{*}\right)\right] x=2\left(L \otimes Q_{j}^{-1}\right) x .
$$

the derivative of the CLQs function is given by

$$
\begin{aligned}
\dot{V}(x)= & \sum_{k=1}^{n_{s}} \tau_{k}(t) 2 x^{T}\left(L \otimes Q_{j}^{-1}\right)\left[\left(I_{n} \otimes A_{k}\right) x\right. \\
& \left.+\left(I_{n} \otimes B_{k}\right)\left(L \otimes Y_{j} Q_{j}^{-1}\right) x\right] \\
& +\sum_{k=1}^{n_{s}} \tau_{k}(t) 2 x^{T}\left(L \otimes Q_{j}^{-1}\right)\left[\left(I_{n} \otimes B_{k}\right)(L \otimes \Delta) x\right] .
\end{aligned}
$$

Noting that $L=U^{T} \Lambda U$, it follows that

$$
\begin{aligned}
& 2 x^{T}\left(L \otimes Q_{j}^{-1}\right)\left[\left(I_{n} \otimes A_{k}\right) x+\left(I_{n} \otimes B_{k}\right)\left(L \otimes Y_{j} Q_{j}^{-1}\right) x\right] \\
& =2 x^{T}\left(U^{T} \otimes I_{p}\right)\left(\Lambda \otimes Q_{j}^{-1}\right)\left(U \otimes I_{p}\right)\left[\left(I_{n} \otimes A_{k}\right) x\right. \\
& \left.\quad+\left(I_{n} \otimes B_{k}\right)\left(U^{T} \otimes I_{p}\right)\left(\Lambda \otimes Y_{j} Q_{j}^{-1}\right)\left(U \otimes I_{p}\right) x\right] .
\end{aligned}
$$

Define $y \triangleq\left(U \otimes I_{p}\right) x$. Eq. (43) can be rewritten in terms of $y$ as

$$
\begin{aligned}
& 2 y^{T}\left(\Lambda \otimes Q_{j}^{-1} A_{k}\right) y+2 y^{T}\left(\Lambda^{2} \otimes Q_{j}^{-1} B_{k} Y_{j} Q_{j}^{-1}\right) y \\
= & \sum_{i=1}^{n} \lambda_{i} y_{i}^{T}\left(2 Q_{j}^{-1} A_{k}+2 \lambda_{i} Q_{j}^{-1} B_{k} Y_{j} Q_{j}^{-1}\right) y_{i} \\
= & \sum_{i=2}^{n} \lambda_{i} y_{i}^{T}\left(2 Q_{j}^{-1} A_{k}+2 \lambda_{i} Q_{j}^{-1} B_{k} Y_{j} Q_{j}^{-1}\right) y_{i}
\end{aligned}
$$

where the last equality is due to the fact that $\lambda_{1}=0$ and Lemma 1. Multiplying (25) from left and right with $Q_{j}^{-1}$ leads to $A_{k}^{T} Q_{j}^{-1}+Q_{j}^{-1} A_{k}+\lambda_{i} Q_{j}^{-1} Y_{j}^{T} B_{k}^{T} Q_{j}^{-1}+$ $\lambda_{i} Q_{j}^{-1} B_{k} Y_{j} Q_{j}^{-1} \leq \sum_{l=1}^{n_{Q}} \eta_{j k l} Q_{j}^{-1}\left(Q_{l}-Q_{j}\right) Q_{j}^{-1}-\beta Q_{j}^{-1}$, $\forall k, j$. It has been proved in Chen et al. (2015) that $\sum_{i=2}^{n} \lambda_{i} y_{i}^{T} Q_{j}^{-1}\left(Q_{l}-Q_{j}\right) Q_{j}^{-1} y_{i} \leq 0$. It thus follows that $\sum_{k=1}^{n_{s}} \tau_{k}(t) 2 x^{T}\left(L \otimes Q_{j}^{-1}\right)\left[\left(I_{n} \otimes A_{k}\right) x+\left(I_{n} \otimes B_{k}\right)(L \otimes\right.$ $\left.\left.Y_{j} Q_{j}^{-1}\right) x\right] \leq-\beta \sum_{i=2}^{n} \lambda_{i} y_{i}^{T} Q_{j}^{-1} y_{i}$. The second term can be analyzed as follows:

$$
\begin{aligned}
& \sum_{k=1}^{n_{s}} \tau_{k}(t) 2 x^{T}\left(L \otimes Q_{j}^{-1}\right)\left[\left(I_{n} \otimes B_{k}\right)(L \otimes \Delta) x\right] \\
= & \sum_{k=1}^{n_{s}} \tau_{k}(t) 2 \sum_{i=2}^{n} \lambda_{i}^{2} y_{i}^{T}\left(Q_{j}^{-1} B_{k} \Delta\right) y_{i} \\
\leq & \sum_{i=2}^{n} 2 \lambda_{i}^{2}\left[\min _{j} \lambda_{\min }\left(Q_{j}\right)\right]^{-1}\left[\max _{k} \sqrt{\lambda_{\max }\left(B_{k}^{T} B_{k}\right)}\right] \bar{e} \bar{\delta} y_{i}^{T} y_{i} \\
\leq & \sum_{i=2}^{n} 2 \lambda_{i}^{2}\left[\min _{j} \lambda_{\min }\left(Q_{j}\right)\right]^{-1}\left[\max _{k} \sqrt{\lambda_{\max }\left(B_{k}^{T} B_{k}\right)}\right] \\
& \times \bar{e} \bar{\delta} \frac{y_{i}^{T} Q_{j}^{-1} y_{i}}{\min _{j}\left[\lambda_{\min }\left(Q_{j}^{-1}\right)\right]} .
\end{aligned}
$$


It follows that $\dot{V}(x) \leq \sum_{i=2}^{n}-\left\{\beta-2 \lambda_{i}\left[\min _{j} \lambda_{\min }\left(Q_{j}\right)\right]^{-1}\right.$ $\left.\max _{k}\left[\sqrt{\lambda_{\max }\left(B_{k}^{T} B_{k}\right)}\right]\left[\min _{j} \lambda_{\min }\left(Q_{j}^{-1}\right)\right]^{-1} \bar{e} \bar{\delta}\right\} \lambda_{i} y_{i}^{T} Q_{j}^{-1} y_{i}$ $\leq-\{\beta-d \bar{e} \bar{\delta}\} V(x)=-\left[\beta-d \bar{e}\left(a+\bar{e} \frac{a b}{1-b \bar{e}}+\frac{c}{1-b \bar{e}}\right)\right] V(x)$. Due to $\bar{e}<\frac{\beta}{\beta b+a d+c d}, \beta-d \bar{e}\left(a+\bar{e} \frac{a b}{1-b \bar{e}}+\frac{c}{1-b \bar{e}}\right)>0$. The proof for the case $x \in E_{j}$ is hence completed. The consensus results can be extended to the cases $x \in \partial O_{V}$ and $x \in \mathbb{R}^{n p}$ by using the same techniques in Chen et al. (2015).

Note that the bound $\frac{\beta}{\beta b+a d+c d}$ of Theorem 2 is a positive constant and can be obtained straightforwardly by solving the BMIs (67) and computing the largest eigenvalue $\lambda_{n}$. Please refer to Tran and Kibangou (2014) for how to estimate the eigenvalues of a Laplacian matrix in a distributed way.

Remark 4 One observation from Theorem 2 is that a $s$ maller value of $\lambda_{n}$ leads to a larger upper bound $\frac{\beta}{\beta b+a d+c d}$ and hence better robustness of the system. Therefore, to get better robustness, the network topology of the multiagent system should be designed in such a way that the largest eigenvalue $\lambda_{n}$ is as small as possible, provided that the connectivity of the network is satisfied. Another observation is that a smaller value of $\max _{k} \sqrt{\lambda_{\max } B_{k}^{T} B_{k}}$ yields a larger upper bound on $\bar{e}$, where $\sqrt{\lambda_{\max } B_{k}^{T} B_{k}}$ is the spectral norm of the matrix $B_{k}$. This is not surprising, because the computation error of $\gamma^{*}$ enters the closed-loop system via the control input matrix $B_{k}, k=1, \ldots, n_{s}$. Similarly, it can be noted that the parameters $Y_{j}$ should be chosen such that $\left[\max _{j} \sqrt{\lambda_{\max }\left(Y_{j}^{T} Y_{j}\right)}\right]$ is as smal$l$ as possible. In addition, as $d \rightarrow 0$, the upper bound $\frac{\beta}{\beta b+a d+c d} \rightarrow \frac{1}{b}=\frac{1}{n_{Q}} \frac{\min _{j} \lambda_{\min }\left(Q_{j}\right)}{\max _{j} \lambda_{\max }\left(Q_{j}\right)}$. In this limit case, we should choose the matrices $Q_{j}$ such that $\min _{j} \lambda_{\min }\left(Q_{j}\right)$ is close to $\max _{j} \lambda_{\max }\left(Q_{j}\right)$. Finally, it is worth mentioning that the choices of the matrices $Y_{j}$ and $Q_{j}$ are all subject to the BMI assumption.

\section{A numerical example}

In the following, a numerical example is presented to verify Theorem 2 . Suppose that the agents are governed by the following second-order linear differential inclusion

$$
\dot{x}_{i}=\sum_{k=1}^{n_{s}} \tau_{k}(t)\left(A_{k} x_{i}+B_{k} u_{i}\right)
$$

where $n_{s}=2, A_{1}=A_{2}=\left[\begin{array}{cc}0 & -1 \\ 1 & 0\end{array}\right], B_{1}=\left[\begin{array}{l}\alpha \\ 1\end{array}\right]$, and $B_{2}=$ $\left[\begin{array}{c}-\alpha \\ 1\end{array}\right]$ with $\alpha=1.05$. The parameters $\tau_{k}(t)$ are generated randomly from the range $(0,1)$ at each time instant, but subject to the constraint $\sum_{k=1}^{n_{s}} \tau_{k}(t)=1$. The Laplacian matrix is given by: $L=\left[\begin{array}{cccc}0.5 & -0.25 & 0 & -0.25 \\ -0.25 & 0.5 & -0.25 & 0 \\ 0 & -0.25 & 0.5 & -0.25 \\ -0.25 & 0 & -0.25 & 0.5\end{array}\right]$, where 0.25 is the weight of each edge. The nonzero eigenvalues of $L$ are $\{0.5,0.5,1\}$. Under the quadratic stabilization framework, the control input $u_{i}$ is given by

$$
u_{i}=\sum_{j=1}^{n} a_{i j} F\left(x_{i}-x_{j}\right)
$$

where $a_{i j}$ is the $(i, j)$ th entry of the adjacency matrix, and the control gain matrix $F$ is determined by a quadratic Lyapunov function. However, it has been shown that for the LDI to be quadratically stabilizable by linear feedback, $\alpha$ should be less than one (Hu (2007)), which indicates that a quadratic Lyapunov function does not exist for consensus of the system (46) under the linear state feedback (47). By solving BMI (25) one obtains the solutions $Q_{1}=\left[\begin{array}{lll}0.6633 & 0.0875 \\ 0.0875 & 0.5649\end{array}\right], Q_{2}=\left[\begin{array}{lll}0.5136 & 0.3388 \\ 0.3388 & 0.6425\end{array}\right]$, $Y_{1}=[0.0357-0.2270], Y_{2}=[0.0805-0.9992], \eta_{111}=0$, $\eta_{112}=8.9404 \times 10^{-4}, \eta_{121}=0, \eta_{122}=0.9990$, $\eta_{211}=0.9992, \eta_{212}=0, \eta_{221}=7.4386 \times 10^{-4}, \eta_{222}=0$, and $\beta=7.9597 \times 10^{-4}$. Thus, Assumption 2 in the paper is satisfied.

For the given Laplacian matrix $L, V(x)$ can be rewritten as $V(x)=\min x^{T}\left[L \otimes\left(\gamma_{1} Q_{1}+\gamma_{2} Q_{2}\right)^{-1}\right] x=$ $\min \sum_{k=1}^{4} \alpha_{k}$, where $\alpha_{1}=0.25\left(x_{1}-x_{2}\right)^{T}\left(\gamma_{1} Q_{1}+\right.$ $\left.\gamma_{2} Q_{2}\right)^{-1}\left(x_{1}-x_{2}\right), \alpha_{2}=0.25\left(x_{2}-x_{3}\right)^{T}\left(\gamma_{1} Q_{1}+\right.$ $\left.\gamma_{2} Q_{2}\right)^{-1}\left(x_{2}-x_{3}\right), \alpha_{3}=0.25\left(x_{3}-x_{4}\right)^{T}\left(\gamma_{1} Q_{1}+\right.$ $\left.\gamma_{2} Q_{2}\right)^{-1}\left(x_{3}-x_{4}\right)$, and $\alpha_{4}=0.25\left(x_{4}-x_{1}\right)^{T}\left(\gamma_{1} Q_{1}+\right.$ $\left.\gamma_{2} Q_{2}\right)^{-1}\left(x_{4}-x_{1}\right)$. The coefficient 0.25 in each $\alpha_{k}$ corresponds to the weight of the edges. Using Algorithm 1, the constructed virtual network topology has 8 virtual nodes, where each virtual node is associated with two variables $v_{\kappa}$ and $w_{\kappa}$. By the finite-time consensus algorithm in Algorithm 1, $v_{\kappa} \rightarrow(1 / 8)$ and $w_{\kappa} \rightarrow \frac{\sum_{k=1}^{4} 2 \alpha_{k}}{8}=\frac{\sum_{k=1}^{4} \alpha_{k}}{4}$. Thus, $\sum_{k=1}^{4} \alpha_{k}$ can be obtained from $\frac{w_{k}}{2 v_{k}}$.

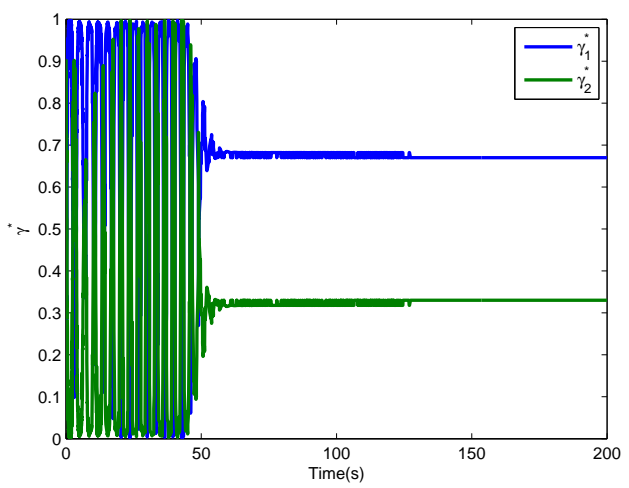

Fig. 1. The optimal parameter $\gamma^{*}$ of the CLQs function

Fig. 1 gives the trajectory of the optimal parameter $\gamma^{*}$ which is obtained by solving the optimization problem 


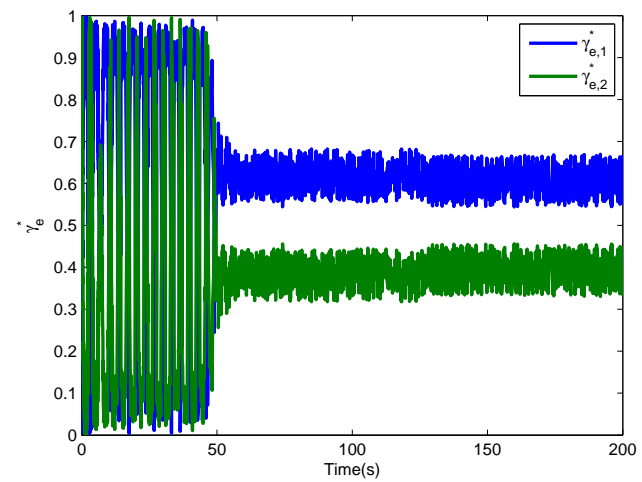

Fig. 2. The perturbed optimal parameter $\gamma_{e}^{*}$

(20)-(22) numerically, where $\gamma_{1}^{*}$ and $\gamma_{2}^{*}$ denote respectively the values of $\gamma^{*}$ in the first and second dimensions. To obtain the disturbed value $\gamma_{e, 1}^{*}$, we add random disturbance $r(t) \times \frac{\beta}{\beta b+a d+c d}$ to $\gamma_{1}^{*}$, where $r(t) \in \mathbb{R}$ is generated randomly from the range $(-1,0)$ whenever $\gamma_{e, 1}^{*}$ is calculated and $\frac{\beta}{\beta b+a d+c d}$ is the bound given in Theorem 2 . The disturbed value $\gamma_{e, 2}^{*}$ is then given by $1-\gamma_{e, 1}^{*}$. The trajectory of the disturbed optimal parameter $\gamma_{e}^{*}$ is given by Fig. 2. It can be observed from Figs. 3 and 4 that a consensus is still achieved, although the disturbed optimal parameter $\gamma_{e}^{*}$ is employed. That is, $\gamma^{*}$ has certain robustness against computational errors (see Theorem 2).

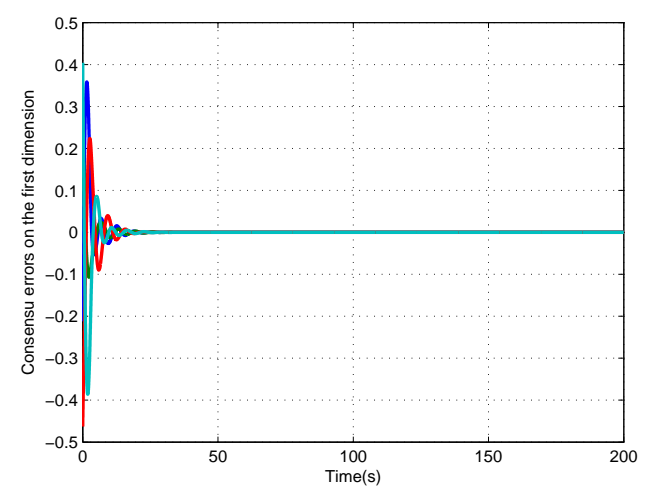

Fig. 3. Consensus errors on the first dimension under randomly generated disturbances

\section{Conclusion}

This paper has focused on the properties of the optimal parameter of the CLQs function. The uniqueness of the optimal parameter has been established. An algorithm has been proposed to compute the optimal parameter in a distributed way. Finally, the robustness issue of the optimal parameter has been investigated for multi-agent

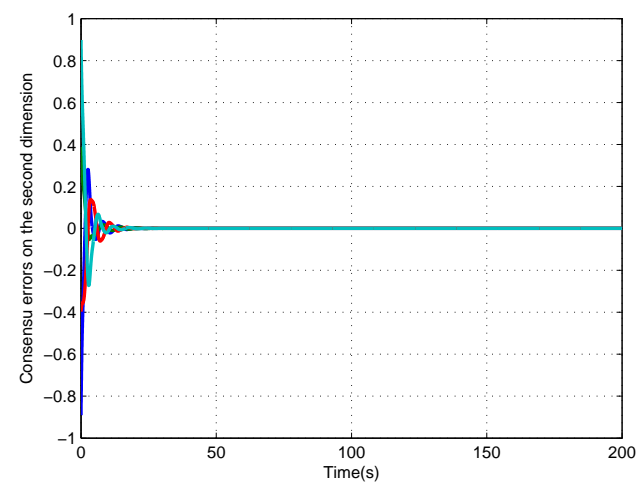

Fig. 4. Consensus errors on the second dimension under randomly generated disturbances

systems described by linear differential inclusions. A numerical example has been provided to validate the theoretical results.

Some future works include: the extension to the directed graph case, application of the CLQs function in controller synthesis of nonlinear multi-agent systems, and use of the CLQs function in event-triggered control.

\section{References}

Boyd, S., L. Ghaoui, E. Feron and V. Balakrishnan (1994). Linear Matrix Inequalities in System and Control Theory. SIAM Studies in Appl. Mathematics. SIAM. Philadelphia.

Boyd, Stephen and Lieven Vandenberghe (2004). Convex optimization. Cambridge university press.

Cao, Y., W. Ren and Z. Meng (2010). Decentralized finite-time sliding mode estimators and their applications in decentralized finite-time formation tracking. Systems \& Control Letters 59(9), 522-529.

Carlone, Luca, Vaibhav Srivastava, Francesco Bullo and Giuseppe C Calafiore (2014). Distributed random convex programming via constraints consensus. SIAM Journal on Control and Optimization 52(1), 629-662.

Chen, F., L. Xiang and W. Ren (2015). Consensus of linear differential inclusions via composite laplacian quadratics. In: American Control Conference. IEEE. pp. 2137-2142.

Chen, F., L. Xiang and W. Ren (2016). Properties of composite laplacian quadratics and their applications in consensus of linear differential inclusions. IEEE Transactions on Automatic Control (doi: 10.1109/TAC.2015.2491739).

Godsil, C. and G. Royle (2001). Algebraic Graph Theory. Springer-Verlag. New York.

Goebel, R., A. R. Teel, T. Hu and Z. Lin (2006). Conjugate convex Lyapunov functions for dual linear differential inclusions. IEEE Transactions on Automatic Control 51(4), 661-666. 
Horn, R. A. and C. R. Johnson (2012). Matrix analysis. Cambridge university press.

Hu, T. (2007). Nonlinear control design for linear differential inclusions via convex hull of quadratics. Automatica 43(4), 685-692.

$\mathrm{Hu}$, T. and Z. Lin (2004). Properties of the composite quadratic Lyapunov functions. IEEE Transactions on Automatic Control 49(7), 1162-1167.

Jadbabaie, A., J. Lin and A. S. Morse (2003). Coordination of groups of mobile autonomous agents using nearest neighbor rules. IEEE Transactions on Automatic Control 48(6), 998-1001.

Liu, R. (1968). Convergent systems. Automatic Control, IEEE Transactions on 13(4), 384-391.

Liu, R., R. Saeks and R. J. Leake (1971). On global linearization. Mathematical Aspects of Electrical Network Analysis 3, 93.

Meng, Z. and Z. Lin (2014). On distributed finite-time observer design and finite-time coordinated tracking of multiple double integrator systems via local interactions. International Journal of Robust and Nonlinear Control 24(16), 2473-2489.

Meng, Z., Z. Zhao and Z. Lin (2013). On global leaderfollowing consensus of identical linear dynamic systems subject to actuator saturation. Systems \& Control Letters 62(2), 132-142.

Moreau, L. (2004). Stability of continuous-time distributed consensus algorithms. In: Decision and Control, 2004. CDC. 43rd IEEE Conference on. Vol. 4. IEEE. pp. 3998-4003.

Moreau, L. (2005). Stability of multi-agent systems with time-dependent communication links. IEEE Transactions on Automatic Control 50(2), 169-182.

Nedic, A. and A. Ozdaglar (2009). Distributed subgradient methods for multi-agent optimization. IEEE Transactions on Automatic Control 54(1), 48-61.

Notarstefano, Giuseppe and Francesco Bullo (2011). Distributed abstract optimization via constraints consensus: Theory and applications. IEEE Transactions on Automatic Control 56(10), 2247-2261.

Olfati-Saber, R. and R. M. Murray (2004). Consensus problems in networks of agents with switching topology and time-delays. IEEE Transactions on Automatic Control 49(9), 1520-1533.

Ooba, T. (2003). On companion systems with state saturation nonlinearity. IEEE Transactions on Circuits and Systems I: Fundamental Theory and Applications 50(12), 1580-1584.

Ren, W. and R. W. Beard (2008). Distributed consensus in multi-vehicle cooperative control. Springer.

Ren, W., R. W. Beard and E. M. Atkins (2007). Information consensus in multivehicle cooperative control: Collective group behavior through local interaction. IEEE Control Systems Magazine 27(2), 71-82.

Sarlette, A. and R. Sepulchre (2009). Consensus optimization on manifolds. SIAM Journal on Control and Optimization 48(1), 56-76.

Sundaram, S. and C. N. Hadjicostis (2007). Finite-time distributed consensus in graphs with time-invariant topologies. In: American Control Conference. IEEE. pp. $711-716$.

Tran, T. D. and A. Y. Kibangou (2014). Distributed estimation of graph laplacian eigenvalues by the alternating direction of multipliers method. In: IFAC World Congress 2014.

Zhu, Minghui and Sonia Martínez (2012). On distributed convex optimization under inequality and equality constraints. IEEE Transactions on Automatic Control 57(1), 151-164. 
Gang Feng received the B.Eng and M.Eng. degrees in Automatic Control from Nanjing Aeronautical Institute, China in 1982 and in 1984 respectively, and the Ph.D. degree in Electrical Engineering from the University of Melbourne, Australia in 1992.

He has been with City University of Hong Kong since 2000 where he is now Chair Professor of Mechatronic Engineering. He was lecturer/senior lecturer at School of Electrical Engineering, University of New South Wales, Australia, 1992-1999. He was awarded an Alexander von Humboldt Fellowship in 1997, and the IEEE Transactions on Fuzzy Systems Outstanding Paper Award in 2007, and Changjiang chair professorship from Education Ministry of China in 2009. His current research interests include multi-agent systems and control, intelligent systems and control, and networked systems and control.

Prof. Feng is an IEEE Fellow, an associate editor of IEEE Trans. Fuzzy Systems and Journal of Systems Science and Complexity, and was an associate editor of IEEE Trans. Automatic Control, IEEE Trans. Systems, Man \& Cybernetics, Part C, Mechatronics, and Journal of Control Theory and Applications. 


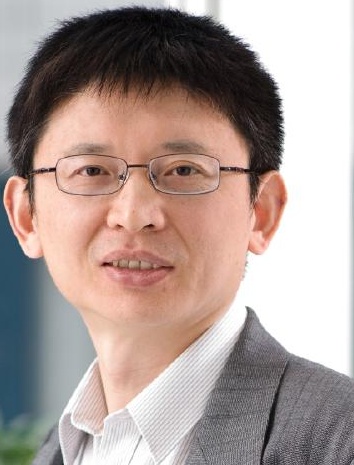


Fei CHEN received his Ph.D. degree in Control Theory and Control Engineering from Nankai University, Tianjin, China, in 2009. From August 2009 to August 2010, he was a Postdoctoral Researcher in the Department of Computer and Electrical Engineering, Utah State University, Logan, UT. Since November 2010, he has been with the Department of Automation, Xiamen University, Xiamen, China, where he is currently an Associate Professor. He was selected as 2014 outstanding reviewer for IEEE Transactions on Control of Network Systems, and awarded New Century Excellent Talents in Fujian Province University in 2015. He is a Senior Member of the IEEE (2015). 


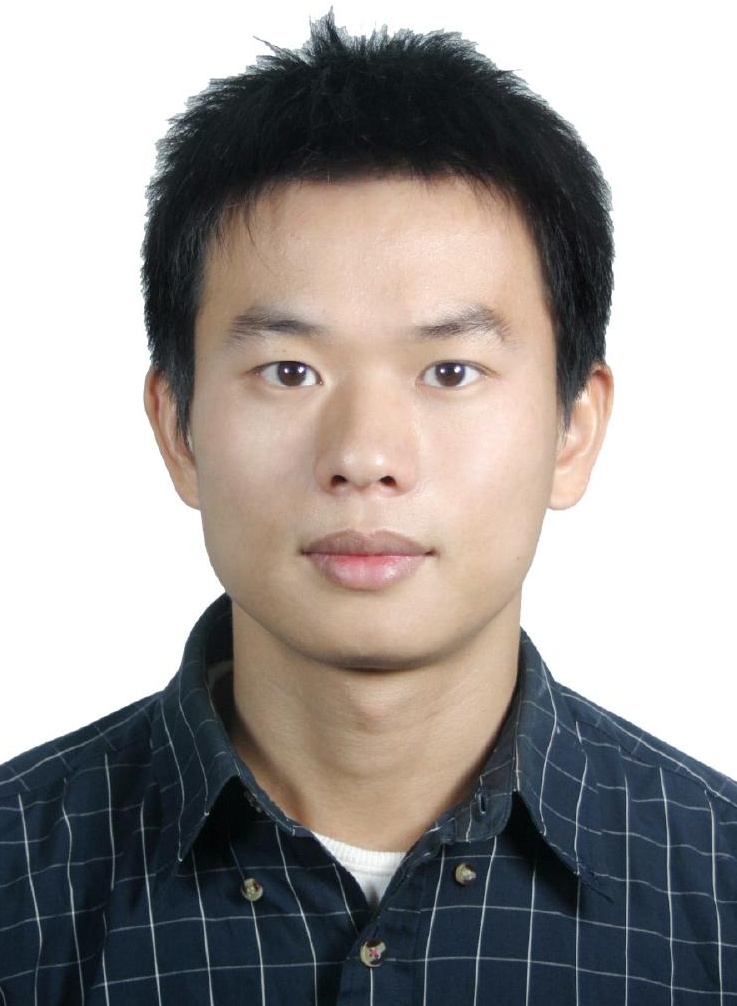

